EPJ Web of Conferences 21, 03004 (2012)

DOI: $10.1051 /$ epjconf/20122103004

(C) Owned by the authors, published by EDP Sciences, 2012

\title{
Inelastic neutron scattering from carbon, iron, yttrium and lead
}

Cecilia Gustavsson ${ }^{\mathrm{a}}$, Jan Blomgren, Carl Hellesen, Angelica Öhrn, Stephan Pomp, and the SCANDAL collaboration

Department of physics and astronomy, Uppsala University, Sweden

\begin{abstract}
Double-differential cross sections and angular distributions of inelastic neutron scattering on ${ }^{12} \mathrm{C},{ }^{56} \mathrm{Fe},{ }^{89} \mathrm{Y}$ and ${ }^{208} \mathrm{~Pb}$ have been measured at $96 \mathrm{MeV}$ at The Svedberg Laboratory, Uppsala, Sweden. Results on elastic neutron scattering at $96 \mathrm{MeV}$ from these nuclei have been reported previously [1-3]. To obtain the inelastic cross sections, a forward-folding technique has been applied. A physically reasonable trial spectrum has been folded with the response function of the detector system and the output has been compared with the experimental data. To create the trial spectrum, a Gaussian has been used for the elastic part and the PRECO code [4-7] for the inelastic part. Other models were tested for the pre-equilibrium contribution and the method was found to be model independent. The response function of the detector setup has been obtained experimentally at the smallest possible angle, in this case at $9 \mathrm{deg}$. The resulting preliminary inelastic scattering data cover an excitation energy range up to $45 \mathrm{MeV}$ and the angular intervals 28 to 58 degrees for ${ }^{12} \mathrm{C}, 26$ to 65 degrees for ${ }^{56} \mathrm{Fe}$ and 26 to 52 degrees for ${ }^{89} \mathrm{Y}$ and ${ }^{208} \mathrm{~Pb}$. The preliminary results are discussed and compared to several model codes as well as existing experimental data for $\left(n, n^{\prime} x\right),\left(n, p^{\prime} x\right)$ and $\left(p, p^{\prime} x\right)$. Possible improvements of the analysis are also discussed.
\end{abstract}

\section{Introduction}

Interactions between a nucleon with low energy (a few $\mathrm{MeV}$ or less) and a nucleus take place mainly with the nucleus as a whole, resulting in the formation of a compound nucleus which subsequently decays, typically by neutron emission. On the other hand, at high incident energies above about 200 $\mathrm{MeV}$, direct nuclear reactions dominate. Pre-equilibrium reactions represent an intermediate process, in which several interactions take place in the nucleus. This results in data with less structure than for direct reactions, but more than for compound reactions.

For compound and direct nuclear reactions, the underlying physics is well understood. Less success has been obtained in the efforts devoted to reaction mechanisms in between compound and direct reactions. One of the reactions where very little data have been reported is the (n,n'x) reaction at intermediate energies. Only one experiment above $30 \mathrm{MeV}$ has been published, reporting data at 65 $\mathrm{MeV}$ from University of California on iron, tin and lead targets [8,9].

An effort was recently undertaken to provide (n,n'x) data at $96 \mathrm{MeV}$ with essentially complete coverage of neutron emission energy. This was performed at The Svedberg Laboratory (TSL) by a Caen-Uppsala collaboration, as part of the EUROTRANS [10] project. In that experiment, part of the SCANDAL [11] setup was used, together with the dedicated converter system CLODIA [12] for neutron detection above $50 \mathrm{MeV}$. The results have however not yet been made available.

This paper presents an another approach to extract information on inelastic neutron emission. A refined and extended data analysis of existing data intended for elastic scattering cross sections only, have been performed and (n,n'x) data for several nuclei and for excitation energies up to $45 \mathrm{MeV}$ in the 25-65 degrees angular range have been extracted.

a e-mail: cecilia.gustavsson@physics.uu.se

This is an Open Access article distributed under the terms of the Creative Commons Attribution-Noncommercial License 3.0, which permits unrestricted use, distribution, and reproduction in any noncommercial medium, provided the original work is properly cited. 


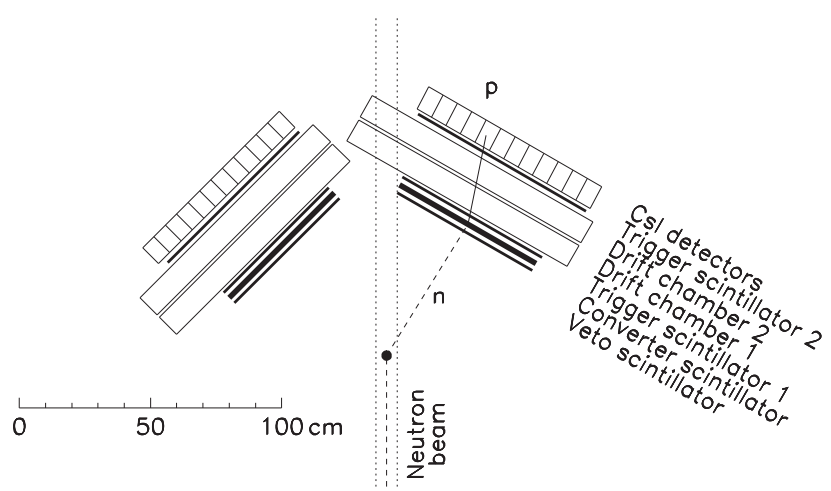

Fig. 1. Schematic layout of the SCANDAL setup. A idealized event is indicated.

\section{Experiment}

Experimental work described in this paper was performed at the neutron beam facility at TSL in Uppsala before the neutron facility was upgraded in 2004. At the then existing neutron facility, a quasi-mono-energetic neutron beam was produced by protons impinging on a ${ }^{7} \mathrm{Li}$ target. The TSL facility and the neutron production has been described in detail in Ref. [11].

For detection of neutrons scattered from a target, the experimental setup SCANDAL (SCAttered Nucleon Detection AssembLy) was used. Fig. 1 presents a layout of this setup which has previously been thoroughly described in Ref. [11]. SCANDAL consists of two identical detector arms positioned on either side of the neutron beam covering angles 10-50 degrees and 30-70 degrees, respectively. Each arm has a thin veto scintillator for charged-particle rejection, a plastic converter scintillator for neutron-proton conversion, a thin $\triangle \mathrm{E}$ scintillator for triggering, two drift chambers (DCHs) for proton tracking, another thin $\Delta \mathrm{E}$ plastic scintillator which is part of the trigger, and an array of CsI detectors (12 on each arm) for energy determination of recoil protons. Hence, the detection of scattered neutrons is based on conversion to protons in an active plastic scintillator and measurement of the full energy of the outgoing protons in an array of CsI detectors.

Prior to the present analysis, our group has published data on elastic neutron scattering from carbon (see Ref. [2]), iron and yttrium (see Ref. [3]) and lead (see Ref. [1]). The preliminary data presented in this paper have been extracted from the same data sets using an analysis procedure based on a forward-folding procedure. The SCANDAL setup has also been used in an experiment measuring double-differential cross sections for light charged particle production, see Ref.[13].

\section{Analysis procedure}

The SCANDAL setup was initially designed for detection of neutrons from elastic scattering events. The energy of the scattered neutron is reconstructed from measuring the energy of conversion protons created in the converter. By measuring the full energy the proton deposits in the CsIs and correcting for energy losses of the proton in the other parts of the detector setup, we obtain the scattered neutron energy.

The converter plastic contains carbon as well as hydrogen, and therefore, neutrons can be converted either by the ${ }^{1} \mathrm{H}(\mathrm{n}, \mathrm{p})$ reaction or the ${ }^{12} \mathrm{C}(\mathrm{n}, \mathrm{p})$ reaction. The $\mathrm{Q}$-value for the ${ }^{12} \mathrm{C}(\mathrm{n}, \mathrm{p})$ reaction is -12.6 $\mathrm{MeV}$ meaning that at forward angles the two conversion reactions can be distinguished by energy difference only. However, the proton energies from the two reactions overlap at angles larger than 20 degrees, and it cannot be decided on an event-by-event basis from which reaction the proton originated. A solution to this problem is to apply an opening angle criterion, demanding that the conversion angle 


\section{$\mathrm{CNR} * 11$}

be smaller than 10 degrees. By taking the value of 10 degrees we take care of the limited experimental resolution in the determination of the conversion angle.

This procedure works well for elastically scattered neutrons which will always have the maximum possible energy when leaving the target. Therefore the design of SCANDAL makes the analysis of elastic neutron scattering events straight-forward, i.e. the elastically scattered neutrons will show up as the events with highest energy after the conversion opening angle cut is made.

Inelastically scattered neutrons, on the other hand, have deposited some of their energy in the scattering event. Therefore, a registered conversion proton, with energy below the elastic scattering maximum, may stem from an inelastic scattering event followed by conversion in a ${ }^{1} \mathrm{H}(\mathrm{n}, \mathrm{p})$ reaction, or from an elastic scattering event followed by conversion in a ${ }^{12} \mathrm{C}(\mathrm{n}, \mathrm{p})$ reaction. We therefore cannot distinguish between these cases on an event-by-event basis and another approach must be used to analyze the data.

\subsection{SCANDAL response function and folding procedure}

We have applied a forward-folding procedure, which is illustrated in Fig. 2. This means that a physically reasonable trial spectrum has been folded with the response function of the experimental setup and the output has been compared to experimental data.

One decisive ingredient in the analysis procedure is the SCANDAL response function, which has several components: Elastically scattered neutrons that are converted to protons through the ${ }^{1} \mathrm{H}(\mathrm{n}, \mathrm{p})$ reaction form a peak at the highest possible energy. In addition, a distribution of protons at lower energies is produced by ${ }^{12} \mathrm{C}(\mathrm{n}, \mathrm{p})$ reactions due to the carbon content in the converter. Another contribution comes from the low-energy tail in the incident neutron beam. Moreover, the response spectrum is slightly distorted by the response of protons in the CsI crystals.

This response function has been studied experimentally in Ref. [11]. In Fig. 10 in Ref. [11], it is shown that the SCANDAL response function can be approximated as the measured $(n, n)$ spectrum at small neutron scattering angles (9 degrees). The reason for this is that, at small angles, elastic scattering dominates heavily meaning that the neutron spectrum hitting SCANDAL is very similar to the incident neutron spectrum impinging onto the scattering target. Therefore, the measured proton energy spectrum (and its corresponding excitation-energy spectrum) obtained in SCANDAL at small angles can be approximated as the response function. With this approach, the response in SCANDAL to incoming neutrons can be described at the energy of the incident beam.

In the preliminary analysis at hand, a measured response function at small neutron scattering angles (9 degrees) has been used. An example of the measured spectrum (i.e. the response function) for the analysis of ${ }^{56} \mathrm{Fe}$ is shown in panel 1 of Fig. 2. To ensure that the contribution from inelastic scattering is negligible at this angle, the TALYS code (see Sect. 4) was used to calculate it at 9 degrees for all nuclei studied. It was found that the contribution from inelastic scattering was less than $1 \%$ of the contribution from elastic scattering which makes the effect negligible.

For the SCANDAL response at lower energies, no measurement was available. It was therefore assumed that the response function at lower energies has the same shape as the one at full energy. This assumption was based on studies of the ratio between the $n p$ scattering and the ${ }^{12} \mathrm{C}(\mathrm{n}, \mathrm{p})$ reaction, which was found to be fairly constant over the relevant energy interval. Hence, the spectrum obtained at $96 \mathrm{MeV}$ (the incident energy) was shifted downwards in steps of $1 \mathrm{MeV}$ in the analysis. The method also assumes the energy resolution in the CsI crystals to be constant. This is not exactly true, but the systematic errors due to variation in energy resolution of the SCANDAL system is far smaller than other uncertainties, and are therefore neglected.

An alternative approach to describing the detector response function would be to model it using e.g. GEANT4, see Ref. [14]. A simulation of the detector response is currently planned in order to verify the procedure.

Panel 2 of Fig. 2 shows the trial spectrum, i.e. a first educated guess. Two components were used as input to the trial spectra; a Gaussian (solid line) to describe the elastic scattering at zero excitation energy, and the PRECO code (see Sect. 4) (dashed line) for the inelastic scattering. Other models were tested for the pre-equilibrium part and it was found that the same correction function was obtained in the end for different trial spectra, as long as the procedure converges to a stable solution. 
EPJ Web of Conferences

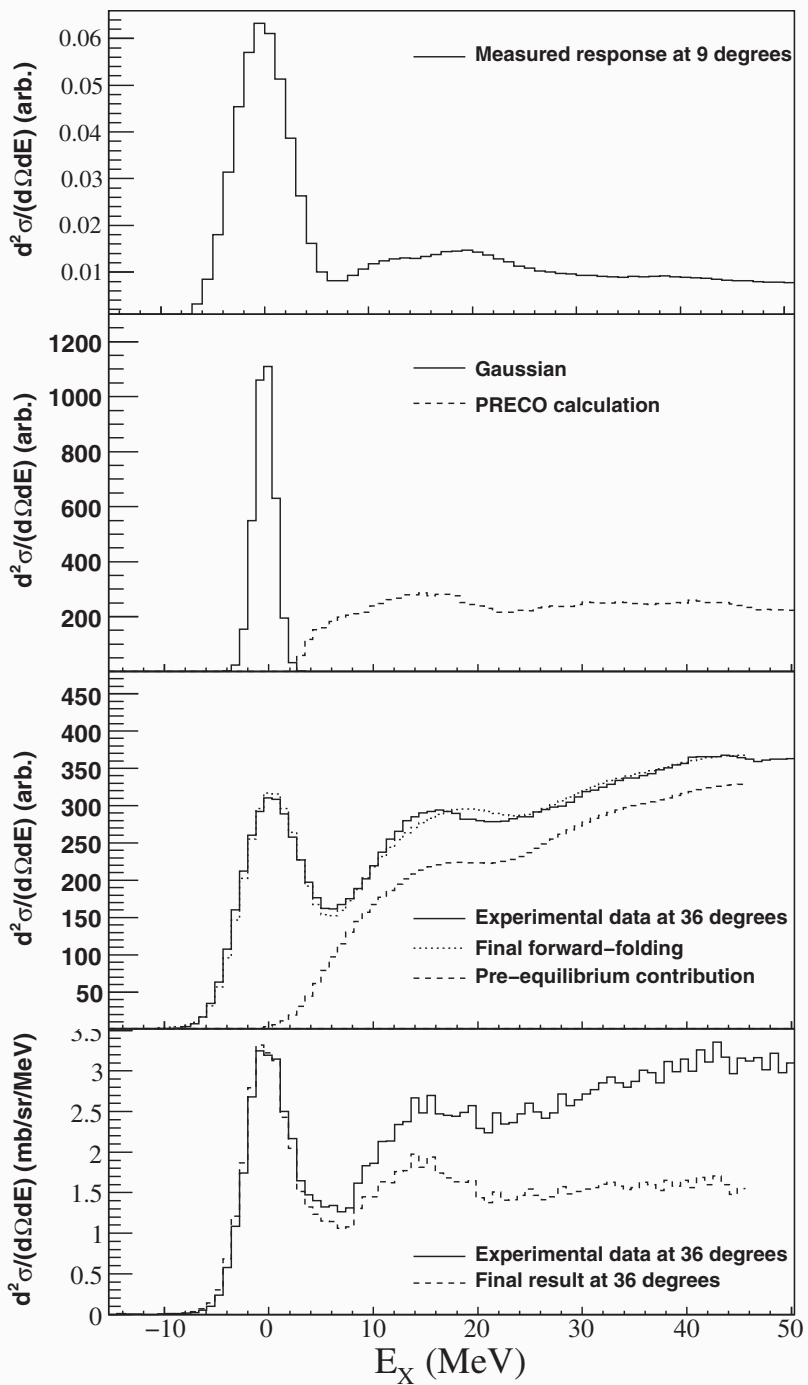

Fig. 2. Analysis procedure. The top panel shows the measured excitation-energy spectrum at 9 degrees corresponding to the response function. The second panels shows the trial spectrum, composed of the ground-state Gaussian (solid line), and the pre-equilibrium prediction (dashed line). The third panel shows the experimental excitation-energy spectrum at 36 degrees (solid histogram). The dotted line is the final forward-folded spectrum, i.e. the total trial spectrum folded with the response after the third iteration. The dashed line is the contribution to the final spectrum coming from the pre-equilibrium prediction. The bottom panel shows the experimental data before (upper histogram, solid) and after (lower histogram, dashed) correction. Also corrections for CsI efficiency and SCANDAL converter efficiency as well as corrections for attenuation and multiple scattering are included.

For the forward-folding, we used an iterative procedure where the difference between the output of the forward-folded trial spectrum and the experimental data was used to modify the input to the next forward-folding. It was found that typically three iterations were required to obtain a stable output. An example is shown in panel 3 of Fig. 2, representing the analysis of ${ }^{56} \mathrm{Fe}$ at 36 degrees. The histogram represents the experimental data and the dotted line is the final forward-folded spectrum, i.e. the total trial spectrum folded with the response after the third iteration. The dashed line is the contribution to the final spectrum coming from the pre-equilibrium prediction. 


\section{$\mathrm{CNR} * 11$}

When the final forward-folded spectrum had been obtained, a ratio between the input and the output of the total forward-folding procedure was used to establish a correction with which the measured spectrum was multiplied bin-by-bin. The result of this correction again for ${ }^{56} \mathrm{Fe}$ at 36 degrees is shown in panel 4 of Fig. 2. The upper histogram (solid) shows the experimental data before corrections and the lower histogram (dashed) after corrections. In this figure, also corrections for CsI efficiency and SCANDAL converter efficiency are included as well as corrections for attenuation and multiple neutron scattering, see Sect. 3.2.

\subsection{Corrections and uncertainties}

In the final steps of the analysis, the energy dependence of the $n p$ cross section was corrected for, as well as variations in proton detection efficiencies in different CsI crystals in the SCANDAL setup. Neutrons can be subjected to attenuation and multiple neutron scattering in the target, and this was corrected for using Monte Carlo techniques, as described in Ref. [3].

The uncertainty in the analysis procedure has been estimated by running one additional step in the iteration of the forward-folded trial spectrum. With one additional step, the description of the experimental spectrum is of equal quality, but slightly different. Thus, the variation in the final result for these two cases should reflect the uncertainty in the spectrum correction procedure.

For the statistical uncertainty, the absolute uncertainty was kept unchanged before and after response correction.

The systematic uncertainties estimated include normalization uncertainties (5\%), uncertainties due to the attenuation and multiple scattering corrections (10\%), effects from the difference in the energy dependence from the $\mathrm{H}(\mathrm{n}, \mathrm{p})$ and the $\mathrm{C}(\mathrm{n}, \mathrm{p})$ reactions $(5 \%)$, and uncertainty in the cross section ratio between the highest and lowest energy in the present analysis (5\%). Assuming all these systematic uncertainties to be uncorrelated, i.e., adding them in quadrature, results in a total systematic uncertainty of $14 \%$. This is an average number in the excitation energy range covered by the present experiment; the uncertainty increases with excitation energy.

\section{Models}

Preliminary data for the for the (n,n'x) reaction are shown in Figs. 3 and 4 together with different models. All model predictions were performed prior to data analysis, i.e. no parameters have been fitted to the data. The models are:

- results from the nuclear reaction code TALYS [15], developed by Koning et al.

- the ICRU63 $[16,17]$ by Chadwick et al. which consists of evaluated data of relevance for neutron and proton cancer therapy applications.

- the nuclear-reaction code HMS-ALICE [18], developed by Blann.

- the code PRECO [4-7] developed by Kalbach. It is mainly used for studying pre-equilibrium reaction mechanisms.

- the quantum molecular dynamics method QMD, which is a microscopic method to calculate nuclear collision processes in the medium to high-energy region [19]. The calculations presented here have been performed by S. Chiba.

- the non-elastic reaction code BRIEFF based on an intra-nuclear cascade model [20,21], developed by Duarte.

\section{Results}

\subsection{Excitation energy}

Fig. 3 shows preliminary excitation energy spectra of the (n,n'x) reaction for ${ }^{56} \mathrm{Fe}$ together with models predictions listed in Sect. 4. The two adjoining panels display the same data but different models for 
clarity. The vertical bars displayed represent the statistical uncertainties only. In this paper, preliminary results for ${ }^{56} \mathrm{Fe}(\mathrm{n}, \mathrm{n}$ 'x) are presented in full, although the total analysis also include inelastic neutron emission up to $45 \mathrm{MeV}$ excitation energy for ${ }^{12} \mathrm{C},{ }^{89} \mathrm{Y}$ and ${ }^{208} \mathrm{~Pb}$.

The excitation-energy spectra show a ground state peak (i.e. elastic scattering) which dominates at small angles and gradually gets smaller with angle. The elastic scattering measurements described have been studied in detail previously [3] and will not be further discussed here. For better statistics, data have been grouped into bins about ten degrees wide. The angles indicated represent the average of that angular bin.

At higher excitation energies all models account reasonably well for the data. At lower excitation energies, some discrepancies are present, but this is expected, since the models presented here do not include a thorough treatment of nuclear structure in the giant resonance region or at lower excitation energies.

In Fig. 3, a giant resonance structure is seen at about $15 \mathrm{MeV}$ excitation energy in data at angles 26 degrees and 36 degrees. The location of this peak is in agreement with the isoscalar quadrupole resonance (ISQR). Giant resonances represent collective excitation modes of nuclei and like discrete nuclear levels they are not modelled in detail in the theory predictions and the resonance is therefore not seen in any of the models.

\subsection{Angular distributions}

Preliminary results on the angular distribution with respect to excitation energy for the (n,n'x) reaction on ${ }^{56} \mathrm{Fe}$ is shown in Fig. 4. It can be seen in the figure that trends in data are in good agreement with model calculations. It is expected that the angular distribution is more forward-peaked at high neutron energies corresponding to low excitation energies. This is seen in Fig. 4 where the upper data set has the most forward-peaked angular distribution and represents the lowest excitation energy $\left(\mathrm{E}_{e x}=5-15\right.$ $\mathrm{MeV}$ ) and hence the highest outgoing neutron energy.

\subsection{Comparison to other reactions}

In Fig. 5, the present preliminary (n,n'x) data for ${ }^{56} \mathrm{Fe}$ and ${ }^{208} \mathrm{~Pb}$ are compared to (n,n'x) data at 65 $\mathrm{MeV}$ for the same nuclei by Hjort et al. [9] from University of California. The shapes are similar, and as expected, the double differential cross section at $96 \mathrm{MeV}$ is lower than at $65 \mathrm{MeV}$. The resonancelike structure can be clearly seen in our data for ${ }^{56} \mathrm{Fe}$ and is seemingly present also in the $65 \mathrm{MeV}$ data set for ${ }^{56} \mathrm{Fe}$.

In Figs. 6 and 7, comparisons are made between the present preliminary (n,n'x) data and (p,p'x) and (n,p'x) data from the literature. Fig. 6 shows data at 26 degrees for ${ }^{56} \mathrm{Fe}$ (upper panel), ${ }^{89} \mathrm{Y}$ (middle panel) and ${ }^{208} \mathrm{~Pb}$ (bottom panel). Fig. 7 shows the same comparisons at 44 degrees. ${ }^{56} \mathrm{Fe}$ data are compared to ${ }^{58} \mathrm{Ni}$ and ${ }^{56} \mathrm{Fe},{ }^{89} \mathrm{Y}$ data are compared to ${ }^{90} \mathrm{Zr}$ data, and ${ }^{208} \mathrm{~Pb}$ data are compared to ${ }^{209} \mathrm{Bi}$ and ${ }^{208} \mathrm{~Pb}$. Angles and energies differ slightly between data sets compared.

In Figs. 6 and 7, filled circles correspond to our data. Open circles correspond to ${ }^{58} \mathrm{Ni}(\mathrm{p}, \mathrm{p}$ 'x) data by Förtsch et al. [22], obtained at $100 \mathrm{MeV}$ incident proton energy. Data are from 1991 and were obtained at the National Accelerator Centre in South Africa. The ${ }^{58} \mathrm{Ni}(\mathrm{p}, \mathrm{p}$ 'x) reaction was studied for several incident proton energies and scattering angles. Data were found to be in reasonably good agreement with previous measurements such as the data from $\mathrm{Wu}$ et al. (see below).

The solid line corresponds to experimental (p,p'x) data from 1979 by Wu et al. [23], which are published at incident proton energy $90 \mathrm{MeV}$ for many nuclei and angles. Raw data are however not available and consequently, the data plotted here have been obtained by extracting data points from a figure in the original published paper.

The filled stars represent (n,p'x) data at $96 \mathrm{MeV}$ for ${ }^{56} \mathrm{Fe}$ and ${ }^{208} \mathrm{~Pb}$ by Blideanu et al. [13]. These data were obtained in 2004 at the $96 \mathrm{MeV}$ neutron beam at TSL using the SCANDAL setup (as well as other detetors). Accordingly, there are many similarities between the experimental setup of this experiments and the ones presented in this paper. 


\section{$\mathrm{CNR} * 11$}

Finally, open squares correspond to ${ }^{209} \mathrm{Bi}(\mathrm{p}, \mathrm{p}$ 'x) data from 1956 by Strauch et al. [24]. In this experiment, energy spectra of protons scattered at 40 degrees were obtained for many different nuclei. The incident proton energy was $96 \mathrm{MeV}$.

In Ref. [9] a comparison between the (n,n'x) and (p,p'x) reaction channels for ${ }^{56} \mathrm{Fe}$ and ${ }^{208} \mathrm{~Pb}$ at $65 \mathrm{MeV}$ showed that the relative strength of the $\left(n, n^{\prime} x\right)$ channel increased with larger mass number $\mathrm{A}$. In that case, the two reaction channels were similar for ${ }^{56} \mathrm{Fe}$ but $(\mathrm{n}, \mathrm{n}$ ' $\mathrm{x})$ more intense for ${ }^{208} \mathrm{~Pb}$. This is an expected result because of the greater neutron number in ${ }^{208} \mathrm{~Pb}$. In our data at 26 degrees shown in Fig. 6, the relative strength of the (n,n'x) reaction channel compared to the the $(n, p$ 'x) channel increases with increasing mass number. For ${ }^{56} \mathrm{Fe}$ (upper panel), the (n,p'x) data are above the (n,n'x) data at high excitation energies, but for ${ }^{208} \mathrm{~Pb}$, the $(\mathrm{n}, \mathrm{n}$ 'x $)$ channel is the strongest.

\section{Outlook and conclusions}

In the presented work, an analysis procedure has been used that allows inelastic neutron emission data to be extracted from an experimental setup optimized for elastic scattering measurements, the SCANDAL system. This procedure involves a forward-folding technique that is based on knowledge of the detector response function, and in the analysis at hand, a measured response function was used. The measurement was however only performed at the highest neutron energy and assumptions were made about the response at lower energies. To also model the response function with a simulation tool like GEANT could be a way of verifying the correctness of using the measured/assumed response function.

Results on the (n,'nx) reaction for ${ }^{12} \mathrm{C},{ }^{56} \mathrm{Fe},{ }^{89} \mathrm{Y}$ and ${ }^{208} \mathrm{~Pb}$ have been obtained. The data presented in this paper are some of the results on ${ }^{56} \mathrm{Fe},{ }^{89} \mathrm{Y}$ and ${ }^{208} \mathrm{~Pb}$. These data are preliminary, but in reasonable agreement with theory predictions, chiefly at higher excitation energies.

Our data are also compared to other available data sets in the literature for the (n,n'x), (n,p'x) and $(p, p$ 'x) reactions and found to be in reasonable agreement.

This work was supported by the Swedish Nuclear Fuel and Waste Management Company, the Swedish Nuclear Power Inspectorate, Ringhals AB, Forsmark AB, the Swedish Defence Research Agency, the Swedish Research Council and the European Council.

The work at Duke University was supported under U.S. Department of Energy grant no. DE-FG02-97ER41033.

\section{References}

1. J. Klug et al., Phys. Rev., C68, 064605(2003).

2. P. Mermod et al., Phys. Rev., C74, 054002(2006).

3. A. Öhrn et al.,Phys. Rev., C77, 24605(2008).

4. C. Kalbach, J. Phys. G: Nucl. Part. Phys., 21, 1519 (1995).

5. C. Kalbach, Phys. Rev., C69, 014605 (2004).

6. C. Kalbach, Phys. Rev., C72, 024607 (2005).

7. C. Kalbach, Phys. Rev., C73, 024614 (2006).

8. E.L. Hjort, et al., Phys. Rev. Lett., 62, 870 (1989).

9. E.L. Hjort, et al., Phys. Rev., C53, 237 (1996).

10. NUDATRA, http://nuklear-server.ka.fzk.de/eurotrans/

11. J. Klug et al., Nucl. Instr. Meth. Phys. Res., A489, 282(2002).

12. I. Sagrado Garcia,et al., in Proceedings of Int. Conf. on Nuclear Data for Science and Technology, Nice, France, 1035 (2008).

13. V. Blideanu, et al., Phys. Rev. C. C70, 014607 (2004).

14. S. Agostinelli et al., Nucl. Instr. Meth. Phys. Res. A506250 (2003).

15. A.J. Koning et al., in Proceedings of the International Conference on Nuclear Data for Science and Technology - ND2007, EDP Sciences, 211 (2008). Code available at http://www.talys.eu/ 
EPJ Web of Conferences

16. ICRU Report 63, Nuclear Data for Neutron and Proton Radiotherapy and for Radiation Protection (International Commission on Radiation Units and Measurements, Bethesda, MD, 2000).

17. M.B. Chadwick et al.Nucl. Sci. Eng., 131, 293 (1999).

18. M. Blann, Phys. Rev., C54, 1341 (1996).

19. J. Aichelin, Phys. Rev., 203, 233 (1991) and references therein.

20. H. Duarte, Phys. Rev., C75, 024611 (2007).

21. H. Duarte, in Proceedings of Int. Conf. on Nuclear Data for Science and Technology, Nice, France, 1117 (2008).

22. S.V. Förtsch, et al., Phys. Rev. C.C43, 691 (1991).

23. J.R. Wu, et al., Phys. Rev. C.C19, 698 (1979).

24. K. Strauch and F. Titus, Phys. Rev. 104, 6191 (1956). 


\section{$\mathrm{CNR} * 11$}

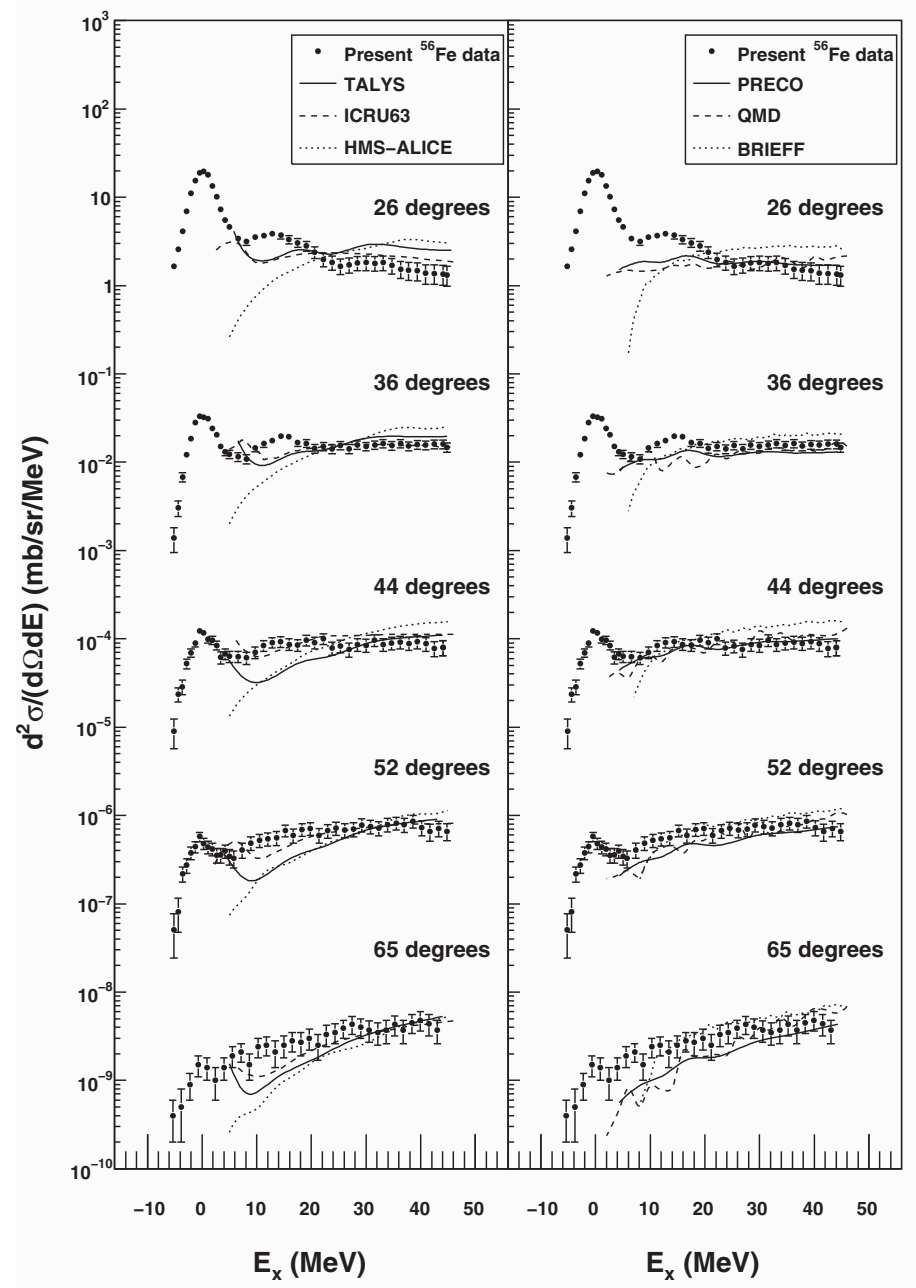

Fig. 3. Preliminary excitation energy spectra of the (n,n'x) reaction on ${ }^{56} \mathrm{Fe}$ at $96 \mathrm{MeV}$ incident neutron energy. From top to bottom, the data points refer to cross sections obtained at an average centre-of-mass angle of 26 , 36 (data multiplied by $10^{-2}$ ), 44 (data multiplied by $10^{-4}$ ), 52 (data multiplied by $10^{-6}$ ) and 65 degrees (data multiplied by $10^{-8}$ ). The error bars display the statistical uncertainties. 


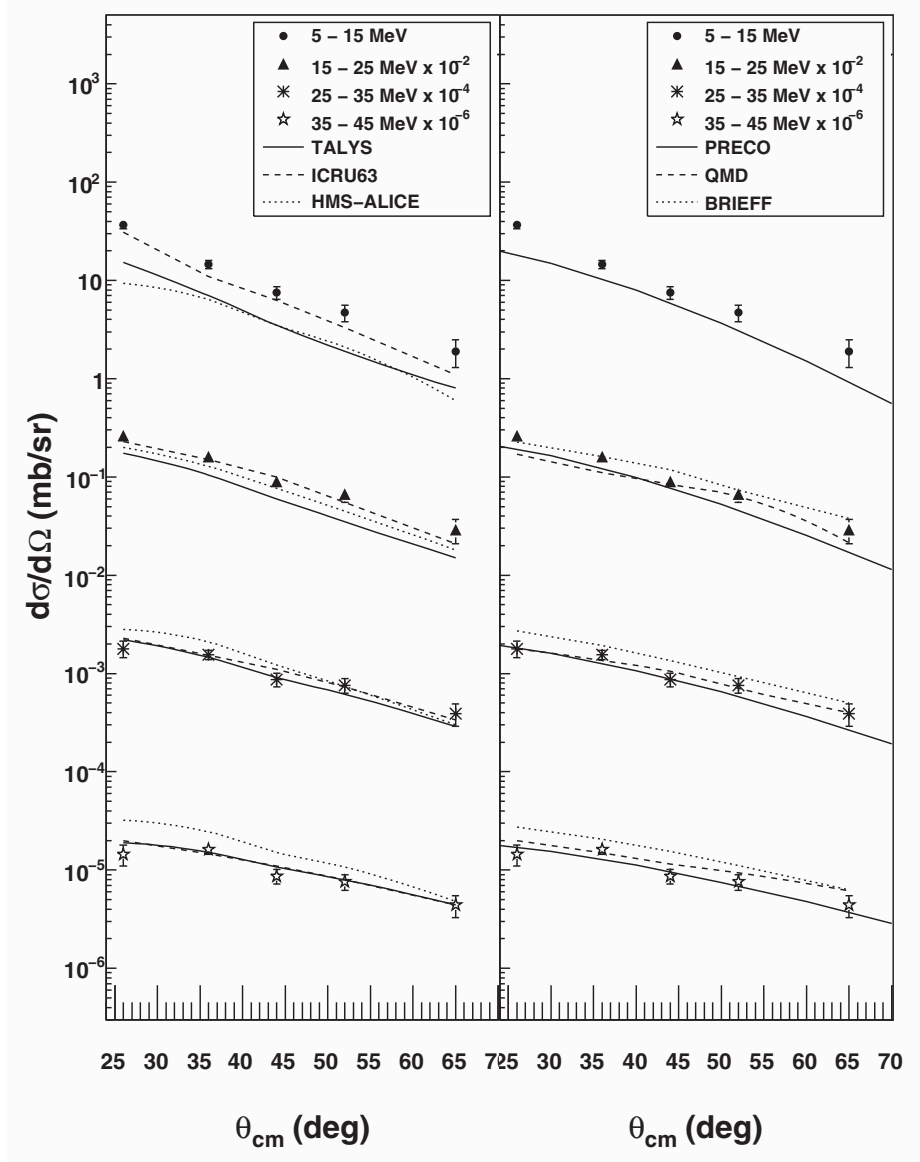

Fig. 4. Preliminary angular distributions with respect to excitation energy for the (n,n'x) reaction on ${ }^{56} \mathrm{Fe}$ at 96 $\mathrm{MeV}$ incident neutron energy. The error bars display the statistical uncertainties. 
$\mathrm{CNR} * 11$

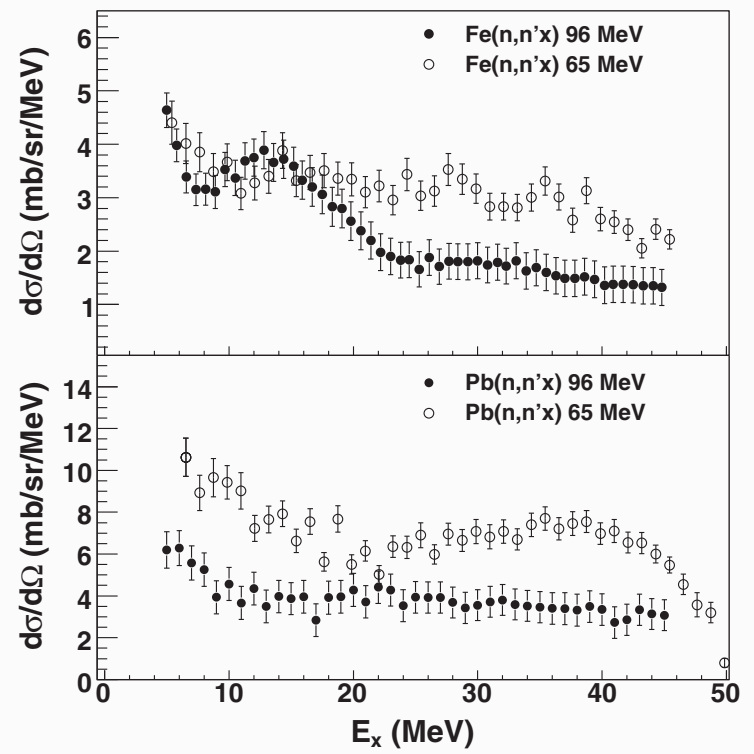

Fig. 5. Comparison of preliminary $96 \mathrm{MeV}{ }^{56} \mathrm{Fe}\left(\mathrm{n}, \mathrm{n}\right.$ 'x) data (upper panel) ${ }^{208} \mathrm{~Pb}(\mathrm{n}, \mathrm{n}$ 'x) and data (lower panel) at 26 degrees (filled circles) with corresponding data from UC Davis at $65 \mathrm{MeV}$ [9] (open circles). 
EPJ Web of Conferences

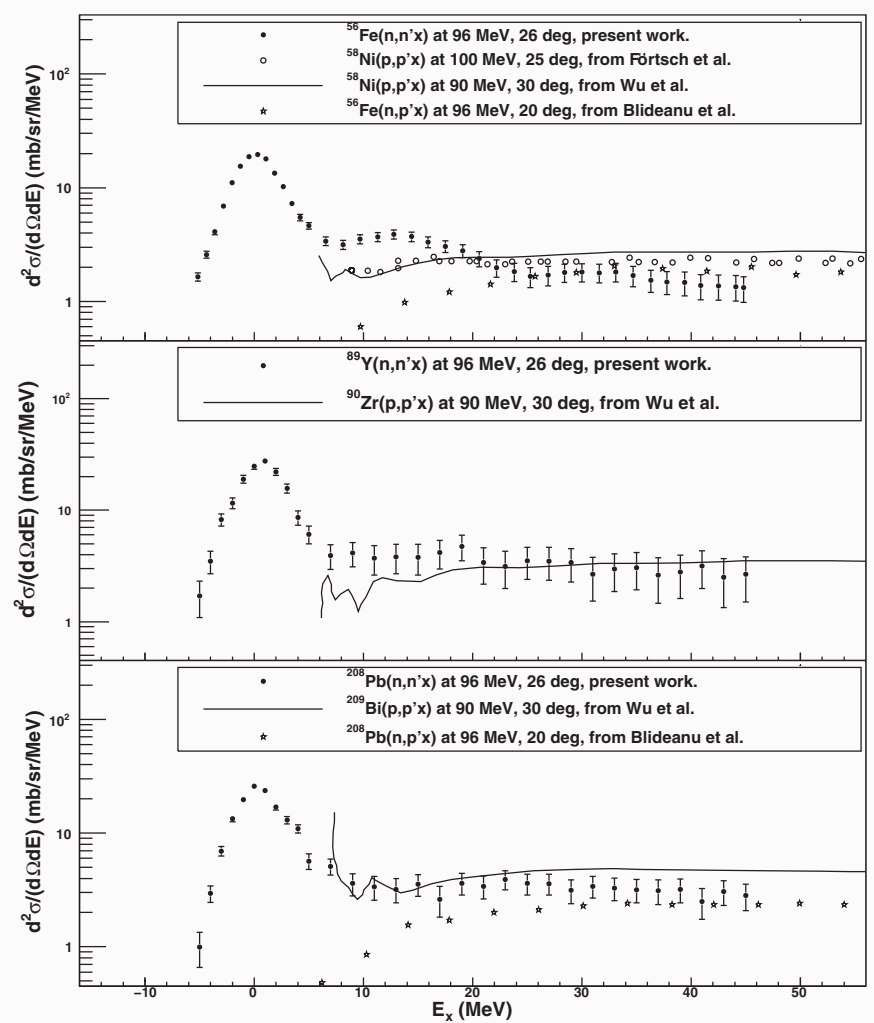

Fig. 6. Comparison of preliminary $96 \mathrm{MeV}$ (n,n'x) data at 26 degrees to (p,p'x) and (n,p'x) data from the literature. The upper panel shows comparison to the ${ }^{56} \mathrm{Fe}(\mathrm{n}, \mathrm{n}$ 'x $)$ data, the middle panel to the ${ }^{89} \mathrm{Y}(\mathrm{n}, \mathrm{n}$ ' $\mathrm{x})$ data and the bottom panel to the ${ }^{208} \mathrm{~Pb}(\mathrm{n}, \mathrm{n}$ 'x $)$ data. Measured angles and energies differ slightly. 
CNR*11

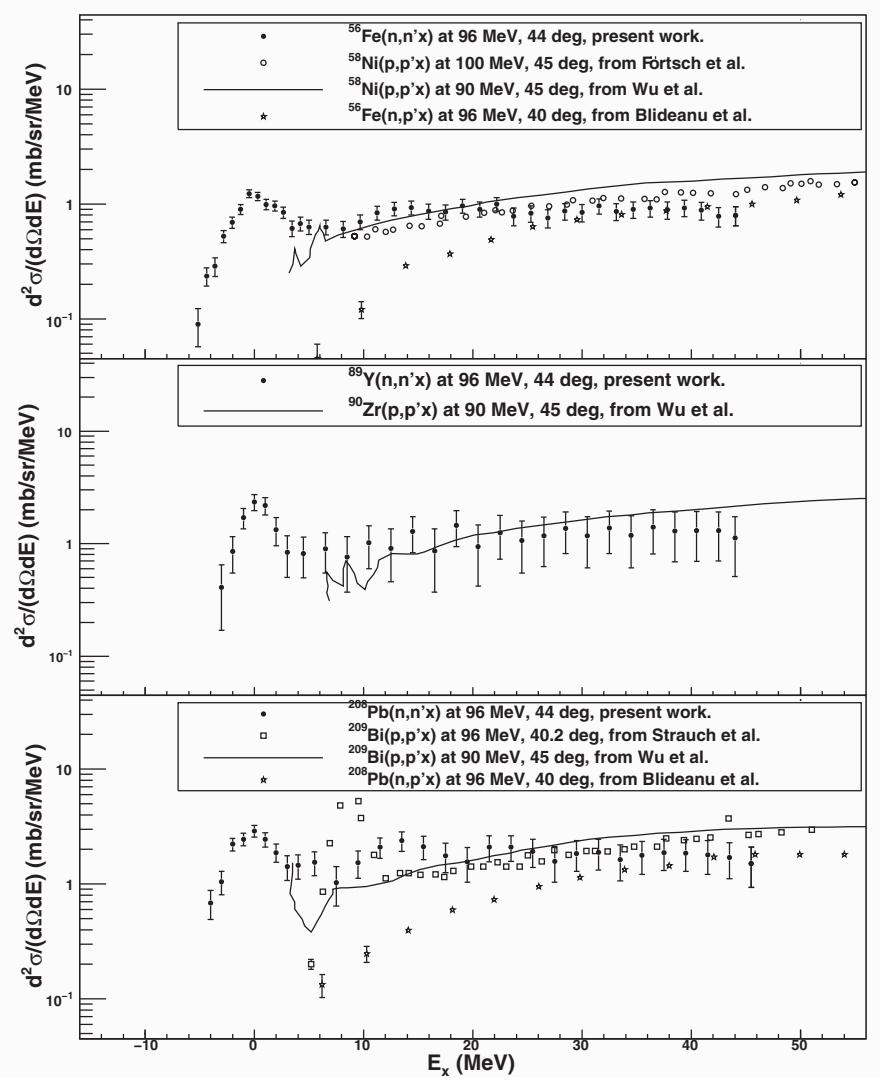

Fig. 7. Comparison of preliminary $96 \mathrm{MeV}\left(n, n^{\prime} x\right)$ data at 44 degrees to (p,p'x) and (n,p'x) data from the literature. The upper panel shows comparison to the ${ }^{56} \mathrm{Fe}(\mathrm{n}, \mathrm{n}$ 'x $)$ data, the middle panel to the ${ }^{89} \mathrm{Y}(\mathrm{n}, \mathrm{n}$ 'x $)$ data and the bottom panel to the ${ }^{208} \mathrm{~Pb}(\mathrm{n}, \mathrm{n}$ 'x $)$ data. Measured angles and energies differ slightly. 